\title{
Management trends for anaplastic meningioma with adjuvant radiotherapy and predictors of long-term survival
}

\author{
Ahmad Alhourani, MD, ${ }^{1}$ Zaid Aljuboori, MD, ${ }^{1}$ Mehran Yusuf, MD, ${ }^{2}$ Shiao Y. Woo, MD, ${ }^{2}$ \\ Eyas M. Hattab, MD, MBA, ${ }^{3}$ Norberto Andaluz, MD, ${ }^{1}$ and Brian J. Williams, MD'
}

Departments of ${ }^{1}$ Neurosurgery, ${ }^{2}$ Radiation Oncology, and ${ }^{3}$ Pathology and Laboratory Medicine, University of Louisville, Louisville, Kentucky

OBJECTIVE The purpose of this study was to describe effects of adjuvant radiotherapy (RT) for anaplastic meningiomas (AMs) on long-term survival, and to analyze patient and RT characteristics associated with long-term survival.

METHODS The authors queried a retrospective cohort of patients with AM from the National Cancer Database (NCDB) diagnosed between 2004 and 2015 to describe treatment trends. For outcome analysis, patients with at least 10 years of follow-up were included, and they were stratified based on adjuvant RT status and propensity matched to controls for covariates. Survival curves were compared. A data-driven approach was used to find a biologically effective dose (BED) of RT with the largest difference between survival curves. Factors associated with long-term survival were quantified. RESULTS The authors identified 2170 cases of AM in the NCDB between 2004 and 2015. They observed increased use of adjuvant RT in patients treated with higher doses. A total of 178 cases met the inclusion criteria for outcome analysis. Forty-five percent $(n=80)$ received adjuvant RT. Patients received a BED of $80.23 \pm 16.6$ Gy (mean \pm IQR). The median survival time was not significantly different (32.8 months for adjuvant RT vs 38.5 months for no RT; $p=0.57$, log-rank test). Dichotomizing the patients at a BED of $81 \mathrm{~Gy}$ showed maximal difference in survival distribution with a decrease in median survival in favor of no adjuvant RT (31.2 months for adjuvant RT vs 49.7 months for no RT; $p=0.03$, log-rank test), but this difference was not significant after false discovery rate correction. Age was a significant predictor for long-term survival.

CONCLUSIONS AMs are aggressive tumors that carry a poor prognosis. Conventional adjuvant RT improves local control. However, the effect of adjuvant radiation on overall survival is unclear. Further investigation into this area is warranted.

https://thejns.org/doi/abs/10.3171/2019.3.FOCUS1960

KEYWORDS anaplastic meningioma; adjuvant radiotherapy; NCDB; National Cancer Database; overall survival

$\mathrm{M}$ ENINGIOMAS remain the most common primary CNS tumors. The vast majority are benign, with less than $2 \%$ harboring malignant histology. ${ }^{14}$ Anaplastic meningiomas (AMs) are associated with a poor clinical course and with high recurrence rates regardless of the extent of resection or adjuvant therapy. ${ }^{1,10}$ Clinical experience remains limited to small case series,${ }^{10}$ leaving several unanswered questions regarding optimal management. Although adjuvant radiotherapy (RT) has demonstrated improved progression-free survival, there is limited evidence regarding its effect on overall survival (OS) ${ }^{1,24}$ and a lack of long-term follow-up data. A large portion of these reports had small patient numbers or appeared prior to the WHO 2000 and 2007 diagnostic criteria updates in which a large portion of WHO grade III tumors were downgraded to WHO grade II. ${ }^{18,23}$ Another controversial point revolves around the optimal dose of RT to administer. There are conflicting reports regarding the optimal dose, with recommended values from $>54 \mathrm{~Gy}^{7,8}$ to $>60 \mathrm{~Gy} ., 16$

We used the National Cancer Database (NCDB) to describe treatment trends for grade III meningioma and the effect of adjuvant RT in AM on long-term survival in a modern cohort following the WHO 2000 update. We hypothesized that adjuvant RT would improve OS.

ABBREVIATIONS AM = anaplastic meningioma; BED = biologically effective dose; IQR = interquartile range; NCDB = National Cancer Database; OS = overall survival PSM = propensity score matching; RT = radiotherapy; RTOG = Radiation Therapy Oncology Group; SRS = stereotactic radiosurgery.

SUBMITTED January 30, 2019. ACCEPTED March 20, 2019.

INCLUDE WHEN CITING DOI: 10.3171/2019.3.FOCUS1960. 
TABLE 1. Trends for treatment of grade III meningiomas from 2004 to 2015

\begin{tabular}{|c|c|c|c|c|}
\hline Year & $\begin{array}{c}\% \\
\text { Receiving } \\
\text { Adjuvant } \\
\text { RT }\end{array}$ & $\begin{array}{c}\text { BED in Gy } \\
\text { (median } \pm \text { IQR) }\end{array}$ & $\begin{array}{c}\% \\
\text { Receiving } \\
\text { Boost } \\
\text { Dose }\end{array}$ & $\begin{array}{c}\% \\
\text { Receiving } \\
\text { SRS }\end{array}$ \\
\hline 2004 & 20.6 & $77.3 \pm 21.3$ & 37.8 & 10.8 \\
\hline 2005 & 25.2 & $80.3 \pm 25.1$ & 36.5 & 2.4 \\
\hline 2006 & 34.5 & $83.1 \pm 17.8$ & 18.9 & 15.5 \\
\hline 2007 & 29.8 & $80.3 \pm 21.2$ & 17.8 & 21.4 \\
\hline 2008 & 30.8 & $80.3 \pm 20.6$ & 20.4 & 10.2 \\
\hline 2009 & 43.4 & $88.3 \pm 19.9$ & 23.5 & 10.6 \\
\hline 2010 & 40.9 & $88.3 \pm 18.5$ & 23.8 & 7.5 \\
\hline 2011 & 36.8 & $88.3 \pm 19.9$ & 19.0 & 6.3 \\
\hline 2012 & 37.4 & $88.3 \pm 20.2$ & 20.0 & 1.5 \\
\hline 2013 & 44.7 & $86.9 \pm 17.5$ & 20.4 & 6.1 \\
\hline 2014 & 45.2 & $88.3 \pm 15.8$ & 14.9 & 9.6 \\
\hline 2015 & 42.8 & $91.9 \pm 12.16$ & 12.9 & 5.2 \\
\hline Linear model $\mathrm{R}^{2}$ & 0.39 & 0.78 & -0.35 & 0.16 \\
\hline Beta coefficient & 0.74 & 0.69 & 0.55 & -0.26 \\
\hline$p$ value & $<0.001$ & $<0.0001$ & $<0.01$ & 0.2 \\
\hline
\end{tabular}

Boldface type indicates statistical significance.

\section{Methods}

We performed a retrospective analysis of AM treatment using the NCDB. The NCDB is a joint project of the Commission on Cancer of the American College of Surgeons and the American Cancer Society. ${ }^{2}$ It captures approximately $70 \%$ of newly diagnosed cancers in the US. The NCDB requires participating facilities to provide follow-up data for at least $90 \%$ of all known living patients annually. ${ }^{12}$ The protocol was approved by the local institutional review board. Informed consent was not required for this study.

The NCDB started collecting information regarding meningiomas in 2004. We identified patients with a diagnosis of meningioma based on the ICD-O-3 (International Classification of Diseases for Oncology, 3rd Edition) by using codes 9530-9539. To describe treatment trends, we included all patients with confirmed WHO grade II meningiomas who underwent resection regardless of missing data points. For outcome analysis, we included only patients with confirmed WHO grade III meningioma who underwent resection with at least 10 years of follow-up data. We excluded patients $<18$ years old, bilateral cases, those with ICD-O-3 topographic codes in the spine or cranial nerves, those receiving neoadjuvant or intraoperative RT, those receiving unknown or unconventional RT modalities, and those with unknown surgical or radiation treatment status. We further excluded patients with 90-day surgery-related mortality to exclude survival bias.

The NCDB started collecting data on the extent of resection for brain locations only after 2009, and does not provide Simpson grading. Therefore, the cohorts were solely stratified by adjuvant RT treatment. We calculated the biologically effective dose (BED) from the total dose and fractionation scheme by using an $\alpha / \beta$ ratio of $3.7 \mathrm{~Gy}$ to standardize dosing across patients. ${ }^{22}$

\section{Statistical Analysis}

Statistical analyses were conducted using MATLAB version 9.3 (The MathWorks, Inc.). Univariate differences were assessed using chi-square tests for categorical covariates and ANOVA for numerical covariates. For trends analysis, a linear regression model was fitted to each predictor variable with the diagnosis year as the response variable. The $\mathrm{R}^{2}$ and $\mathrm{p}$ values describing the fit of the model are reported. We adjusted for covariates when comparing survival with and without adjuvant RT by using propensity score matching (PSM). We matched patients who received adjuvant RT in a 1:1 fashion to balance age, sex, race, tumor size, and Charlson-Deyo comorbidity score. ${ }^{4}$ Survival distributions were compared using the log-rank test and plotted using Kaplan-Meier plots. A Cox proportional hazards model was applied to delineate factors associated with long-term survival. All tests were 2-sided, and a $\mathrm{p}$ value $<0.05$ was considered statistically significant.

Adjuvant RT dose effect on survival was assessed through serially dichotomizing the adjuvant RT cohort into high- and low-dose cohorts at different BED thresholds from 65 to $100 \mathrm{~Gy}$ in steps of $2 \mathrm{~Gy}$ and comparing the survival distribution between the two cohorts using a log-rank test. A false discovery rate of 5\% was applied to the $\mathrm{p}$ values to correct for the multiple comparisons.

\section{Results}

We identified 2170 cases of grade III meningioma treated with resection between 2004 and 2015. We observed a trend for increased utilization of adjuvant RT (from 20.6\% of the cases in 2004 to $42.8 \%$ of the cases in 2015) with higher BEDs (from 77.3 \pm 21.3 Gy in 2004 to $91.9 \pm 12.16$ Gy in 2015; median \pm interquartile range [IQR]). Interestingly, we observed decreased utilization of boost dosesfrom $37.8 \%$ of the cases in 2004 to $12.9 \%$ of the cases in 2015-with no change in the trend of stereotactic radiosurgery (SRS) utilization (Table 1).

Of those 2170 cases, 178 patients between 2004 and 2006 met our inclusion criteria and had 10-year follow-up data. Forty-five percent $(n=80)$ received adjuvant RT. To adjust for potential confounders, we used PSM to match 72 patients from each treatment group. Baseline characteristics were not significantly different after matching (Table 2).

\section{Adjuvant RT Characteristics}

Patients received adjuvant RT on average at $53.3 \pm 33.9$ days (mean \pm SD) from surgery. Patients received a BED of $80.23 \pm 16.6$ Gy (median \pm IQR), which is equivalent to $54 \mathrm{~Gy}$ in 30 fractions. Only 16 patients received a boost dose following their initial adjuvant RT, and SRS was used in $8.8 \%(\mathrm{n}=7 / 80)$ of the cases.

\section{Survival Analysis With Adjuvant RT}

Kaplan-Meier survival curves for the unmatched cohorts were not significantly different $(\log$-rank test, $\mathrm{p}=$ 0.57 ) after eliminating patients with 90 -day mortality who 
TABLE 2. Demographic and clinical characteristics of AM in both the unmatched and matched cohorts

\begin{tabular}{|c|c|c|c|c|c|c|c|c|c|c|c|c|}
\hline \multirow[b]{3}{*}{ Variable } & \multicolumn{6}{|c|}{ Unmatched Cohort } & \multicolumn{6}{|c|}{ Matched Cohort } \\
\hline & \multicolumn{2}{|c|}{$\begin{array}{c}\text { Op Only, } \\
\mathrm{n}=98\end{array}$} & \multicolumn{2}{|c|}{$\begin{array}{c}\text { Op w/ Adjuvant RT, } \\
n=80\end{array}$} & \multirow[b]{2}{*}{$\chi^{2} /$ F-stat } & \multirow[b]{2}{*}{$p$ Value } & \multicolumn{2}{|c|}{$\begin{array}{c}\text { Op Only, } \\
\mathrm{n}=72\end{array}$} & \multicolumn{2}{|c|}{$\begin{array}{c}\text { Op w/ Adjuvant RT, } \\
n=72\end{array}$} & \multirow[b]{2}{*}{$\chi^{2 / F-s t a t ~}$} & \multirow[b]{2}{*}{$p$ Value } \\
\hline & No. & $\%$ & No. & $\%$ & & & No. & $\%$ & No. & $\%$ & & \\
\hline \multicolumn{13}{|l|}{ Race } \\
\hline White & 74 & 76.29 & 63 & 80.77 & 0.51 & 0.47 & 58 & 80.56 & 57 & 79.17 & 0.04 & 0.84 \\
\hline Nonwhite & 23 & 23.71 & 15 & 19.23 & & & 14 & 19.44 & 15 & 20.83 & & \\
\hline \multicolumn{13}{|l|}{ Sex } \\
\hline Male & 48 & 48.98 & 35 & 43.75 & 0.48 & 0.49 & 31 & 43.06 & 34 & 47.22 & 0.25 & 0.62 \\
\hline Female & 50 & 51.02 & 45 & 56.25 & & & 41 & 56.94 & 38 & 52.78 & & \\
\hline \multicolumn{13}{|l|}{ Facility type } \\
\hline Research & 47 & 51.09 & 40 & 52.63 & 3.33 & 0.19 & 34 & 48.57 & 31 & 46.97 & 3.16 & 0.21 \\
\hline Community & 31 & 33.70 & 31 & 40.79 & & & 31 & 44.29 & 24 & 36.36 & & \\
\hline Network & 14 & 15.22 & 5 & 6.58 & & & 5 & 7.14 & 11 & 16.67 & & \\
\hline \multicolumn{13}{|l|}{ Charlson-Deyo score } \\
\hline 0 & 71 & 72.45 & 64 & 80.00 & 2.02 & 0.57 & 56 & 77.78 & 56 & 77.78 & 0.24 & 0.97 \\
\hline 1 & 19 & 19.39 & 13 & 16.25 & & & 13 & 18.06 & 12 & 16.67 & & \\
\hline 2 & 6 & 6.12 & 2 & 2.50 & & & 2 & 2.78 & 3 & 4.17 & & \\
\hline$\geq 3$ & 2 & 2.04 & 1 & 1.25 & & & 1 & 1.39 & 1 & 1.39 & & \\
\hline \multicolumn{13}{|l|}{ Tumor size } \\
\hline$\leq 3 \mathrm{~cm}$ & 8 & 8.16 & 7 & 8.75 & 0.02 & 0.89 & 6 & 8.33 & 6 & 8.33 & 0.12 & 0.73 \\
\hline$>3 \mathrm{~cm}$ & 90 & 91.84 & 73 & 91.25 & & & 66 & 91.67 & 66 & 91.67 & & \\
\hline Median age, yrs (IQR) & 67 & $(22.00)$ & 67 & $(22.00)$ & 0.12 & 0.73 & 67 & $(21.50)$ & 64 & $(25.50)$ & 0.25 & 0.62 \\
\hline
\end{tabular}

F-stat $=$ F-statistic .

With the exception of age, which is expressed as the median (IQR), all clinical and demographic variables are expressed as the number of patients and percent. There are differences in totals due to missing data for some of the variables.

would potentially have received RT. The median survival with adjuvant RT was 32.8 months compared to 38.5 months without adjuvant RT. Correcting for covariates by using PSM showed similar results, with a median survival of 33.5 months for patients receiving adjuvant RT compared to 38.5 months without RT (log-rank test, $\mathrm{p}=0.5$ ), as depicted in Fig. 1.

We serially dichotomized the adjuvant RT cohort at different BED thresholds into low- and high-dose RT. The maximal statistical difference in survival was reached at a BED of 81 Gy (log-rank test, $p=0.03$ ). Counterintuitively, it showed improved survival for the lower-dose RT group, with a median survival of 49.7 months compared to 31.2 months for patients receiving a higher dose (Fig. 2). This difference was not significant after correcting for multiple comparisons (see above).

Finally, we analyzed factors associated with long-term survival ( $>10$ years). On univariate analysis, survivors were significantly younger (median $\pm \mathrm{IQR}$ ): $54 \pm 22.2$ years versus $69 \pm 20.5$ years (F-statistic 13.44, p < 0.001). Using a Cox proportional hazards model, age remained a significant predictor of survival. Additionally, tumor size and a Charlson-Deyo comorbidity score $\geq 3$ were independent predictors of mortality (Table 3 ).

\section{Discussion}

We used the NCDB to describe treatment trends for
AM in which more aggressive treatment was used upfront. We also describe the effect of adjuvant RT for AM on long-term survival by using a propensity-matched cohort. Contrary to our hypothesis, adjuvant RT did not confer any long-term survival benefit. We also did not observe a survival benefit with higher doses. Finally, long-term survival was associated with younger age.

Analyzing treatment trends, we observed a trend toward more aggressive first-line treatment, with more patients receiving adjuvant $\mathrm{RT}$ with higher radiation doses. This translated into fewer patients requiring boost dose treatments. This reflects the current understanding of the aggressive nature of the disease and its resistance to most adjuvant treatment. Interestingly, we did not observe any change in the utilization of SRS for RT administration. To our knowledge, this is the first report on the treatment of AM that includes long-term survival data at 10 years and in which OS with and without adjuvant RT is compared. One report presented long-term OS at 10 years but included atypical meningiomas in the cohort. ${ }^{16}$ Only a handful of reports compared OS with or without adjuvant RT. A report by Yang et al. ${ }^{24}$ showed a minimal effect for adjuvant RT on survival, with an OR of 0.006 in a cohort of 24 patients. Similarly, Zhao et al. ${ }^{25}$ found a modest benefit for RT on OS in a series of 37 patients, with a hazard ratio of 0.15 . One study showed a trend for a survival benefit (OS 5.4 vs 2.5 years) in patients who received adjuvant RT ( $\mathrm{n}=$ 3 ) versus those who did not $(n=10)$; however, there was an 

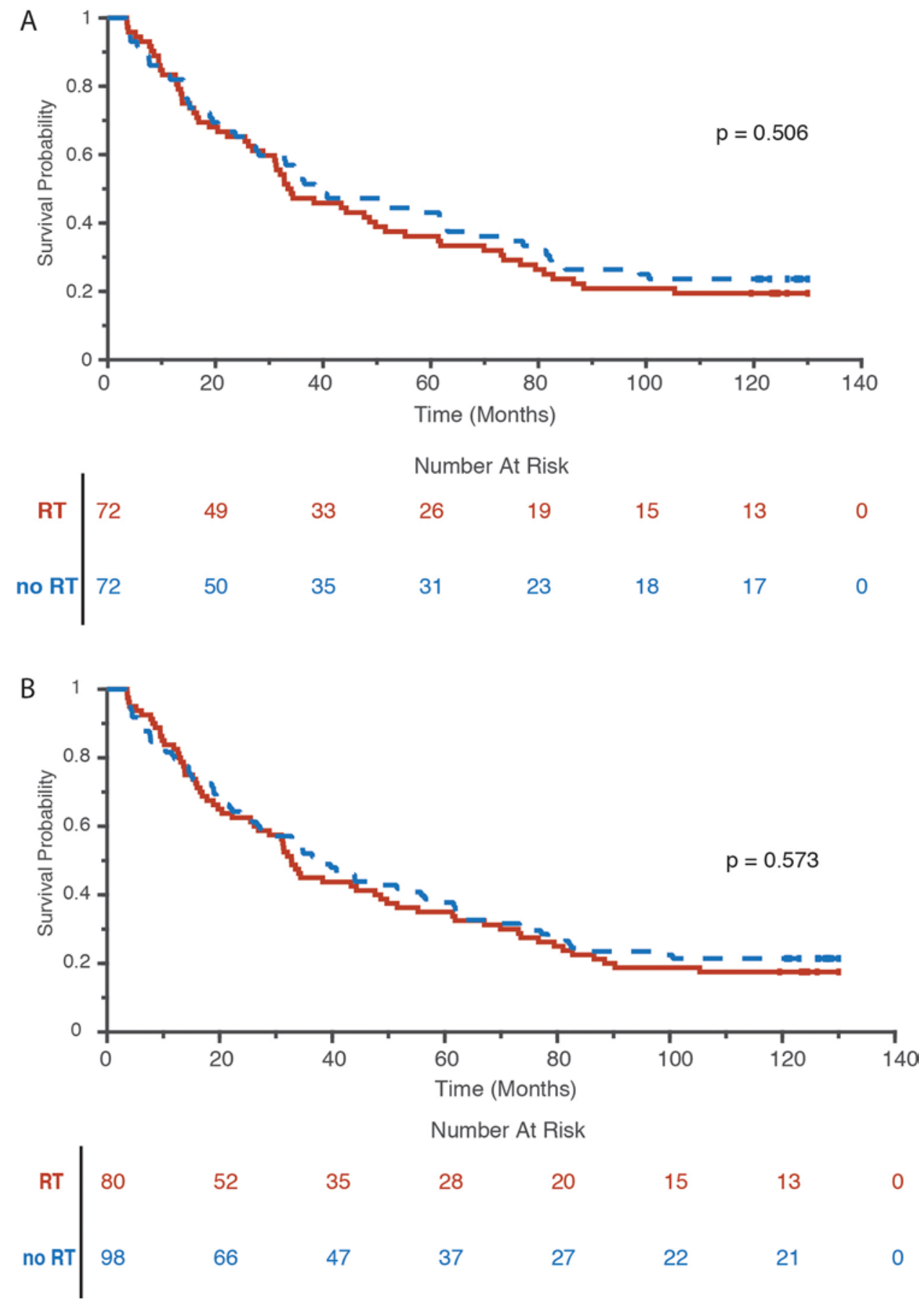

FIG. 1. Kaplan-Meier OS plots for patients with AM who underwent adjuvant RT versus no adjuvant RT for the matched cohort (A) and the unmatched cohort (B). p value from log-rank test is shown. The y-axis represents survival probability.

18 -year difference in the mean age of the groups (50.3 vs 68.5 years, respectively). ${ }^{18}$ The largest cohort was using the NCDB and showed improved OS at 5 years with adjuvant RT. ${ }^{13}$ However, it included patients with 90-day surgeryrelated mortality, which can lead to survival bias..$^{5} \mathrm{We}$ replicated the findings and show no difference in survival after excluding those patients (Fig. 3). Several case series did not observe any difference in OS following RT. ${ }^{6,18-20,24}$ Additionally, several reports in which the analysis of the
Surveillance, Epidemiology, and End Results (SEER) database was used did not show improved survival following adjuvant RT. ${ }^{1,19}$ Although these results are disappointing, they indicate the need for different management strategies in dealing with these aggressive tumors.

There is continued controversy regarding the optimal dosing for AM. Given this uncertainty we used a datadriven approach to find a dosing effect on survival. We found a detrimental effect for RT dosing above a BED of 


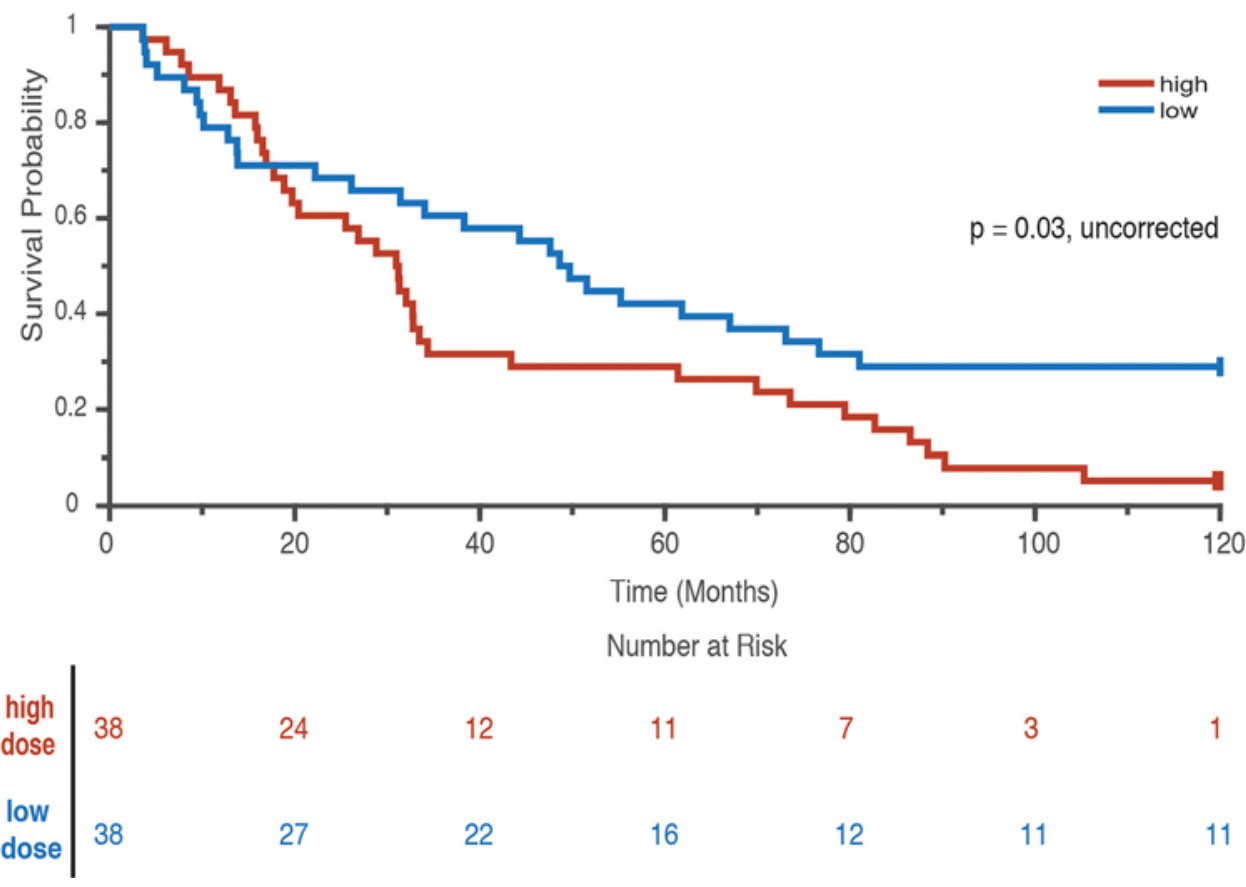

FIG. 2. Kaplan-Meier OS plot for patients with AM who underwent adjuvant RT comparing high- and low-dose RT at a BED threshold of $81 \mathrm{~Gy}$. p value from log-rank test is shown. The y-axis represents survival probability.

TABLE 3. Univariate and multivariate Cox proportional hazards model for survival

\begin{tabular}{|c|c|c|c|c|c|c|c|c|c|c|}
\hline \multirow[b]{3}{*}{ Variable } & \multicolumn{6}{|c|}{ Univariate } & & & & \\
\hline & \multicolumn{2}{|c|}{ Dead } & \multicolumn{2}{|c|}{ Alive } & \multirow[b]{2}{*}{$\chi^{2} /$ F-stat } & \multirow[b]{2}{*}{$p$ Value } & \multicolumn{4}{|c|}{ Multivariate } \\
\hline & No. & $\%$ & No. & $\%$ & & & Beta & p Value & Hazard Ratio & $95 \% \mathrm{Cl}$ \\
\hline \multicolumn{11}{|l|}{ Race } \\
\hline White & 110 & 78.57 & 27 & 77.14 & 0.03 & 0.85 & Reference & & & \\
\hline Nonwhite & 30 & 21.42 & 8 & 22.85 & & & -0.04 & 0.85 & 0.95 & $0.609-1.51$ \\
\hline \multicolumn{11}{|l|}{ Sex } \\
\hline Male & 70 & 48.95 & 13 & 37.14 & 1.58 & 0.2 & Reference & & & \\
\hline Female & 73 & 51.04 & 22 & 62.85 & & & -0.23 & 0.21 & 0.79 & $0.55-1.14$ \\
\hline \multicolumn{11}{|l|}{ Facility type } \\
\hline Research & 71 & 52.98 & 16 & 47.05 & 1.01 & 0.6 & Reference & & & \\
\hline Community & 47 & 35.07 & 15 & 44.11 & & & -0.23 & 0.25 & 0.79 & $0.54-1.18$ \\
\hline Network & 16 & 11.94 & 3 & 8.82 & & & 0.11 & 0.71 & 1.12 & $0.62-2.02$ \\
\hline \multicolumn{11}{|l|}{ Charlson-Deyo score } \\
\hline 0 & 108 & 75.52 & 27 & 77.14 & 1.1 & 0.77 & Reference & & & \\
\hline 1 & 25 & 17.48 & 7 & 20.00 & & & 0.12 & 0.61 & 1.13 & $0.71-1.79$ \\
\hline 2 & 7 & 4.89 & 1 & 2.85 & & & 0.44 & 0.27 & 1.56 & $0.71-3.44$ \\
\hline$\geq 3$ & 3 & 2.09 & 0 & 0 & & & 1.72 & 0.01 & 5.61 & $1.65-19.11$ \\
\hline \multicolumn{11}{|l|}{ Tumor size } \\
\hline$\leq 3 \mathrm{~cm}$ & 10 & 6.993 & 5 & 14.28 & 1.94 & 0.16 & Reference & & & \\
\hline$>3 \mathrm{~cm}$ & 133 & 93.00 & 30 & 85.71 & & & 0.81 & 0.03 & 2.24 & $1.07-4.7$ \\
\hline Median age, yrs (IQR) & 69 & $(20.5)$ & 54 & $(22.25)$ & 13.44 & $<0.001$ & 0.04 & $<0.001$ & 1.04 & $1.02-1.05$ \\
\hline
\end{tabular}

With the exception of age, which is expressed as the median (IQR), all clinical and demographic variables are expressed as the number of patients and \%. There are differences in totals due to missing data for some of the variables. Boldface type indicates statistical significance. 


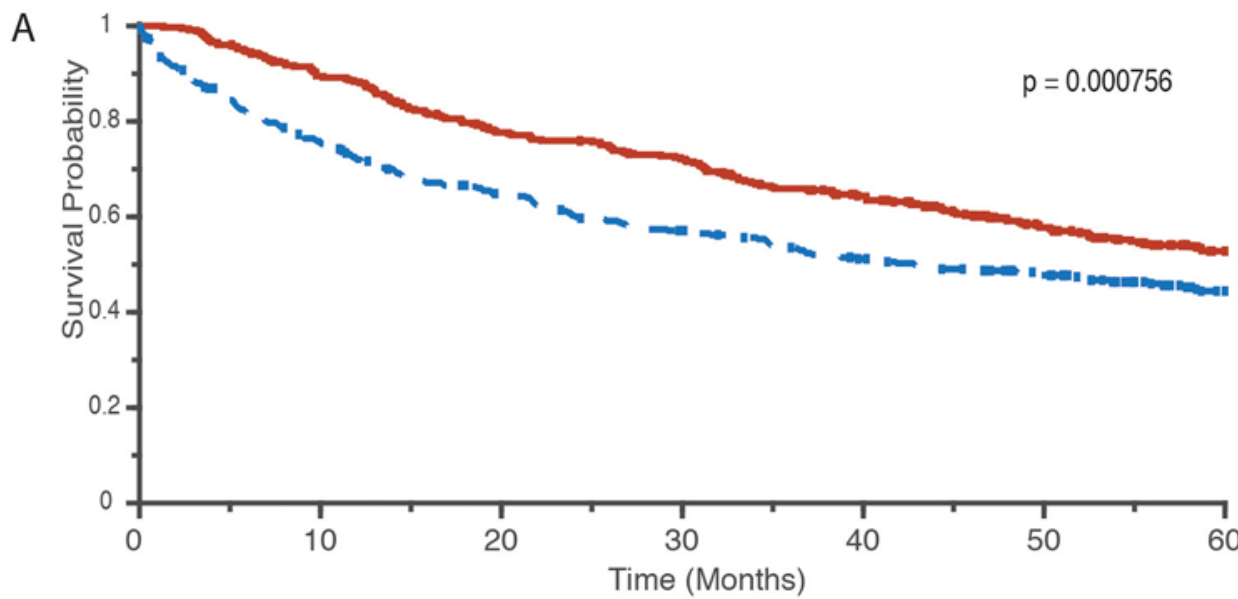

\begin{tabular}{r|rrrrrr}
\multicolumn{5}{c}{ Number At Risk } \\
RT & & & \multicolumn{7}{c}{121} \\
353 & 313 & 267 & 246 & 206 & 163 & 110
\end{tabular}

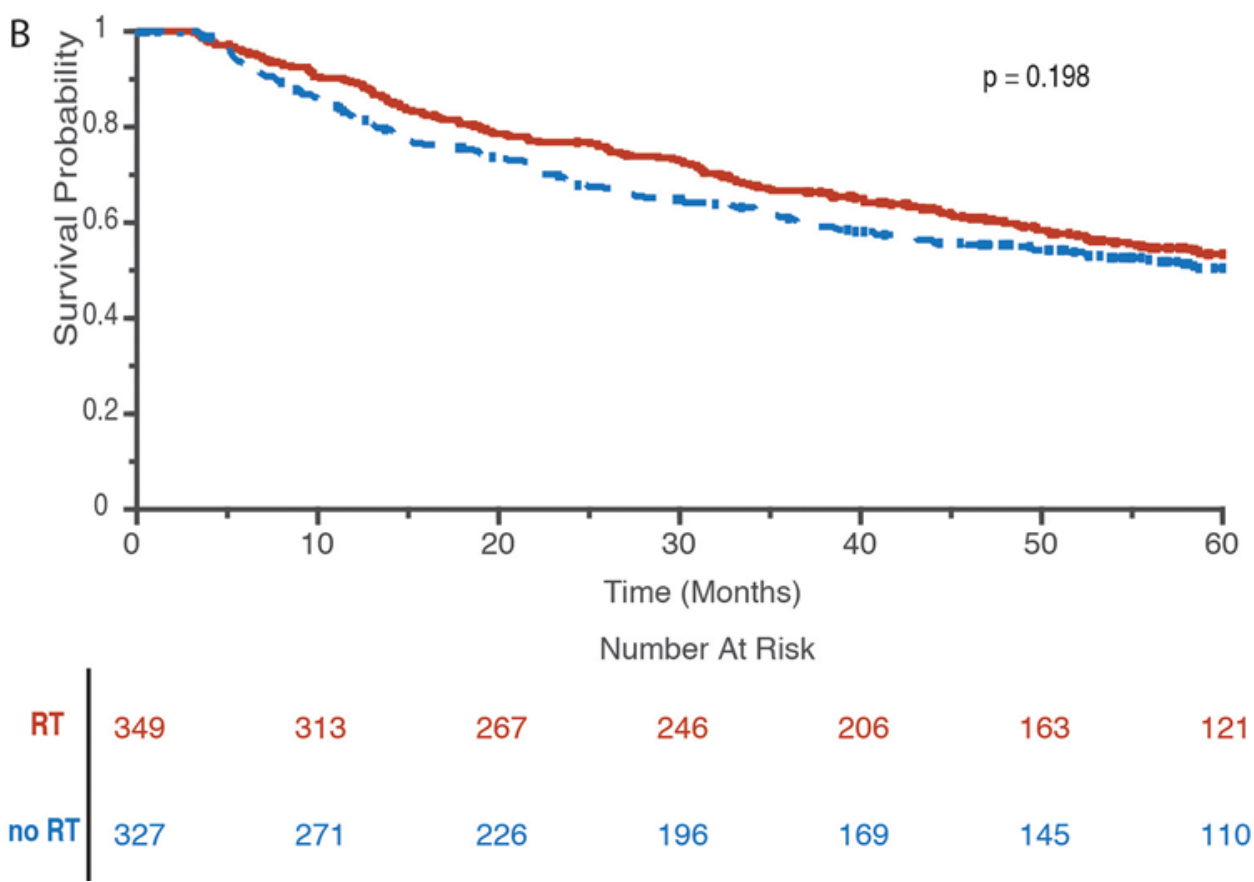

FIG. 3. Kaplan-Meier OS plots for patients with AM who underwent adjuvant RT vs no adjuvant RT between 2004 and 2012 , with patients who died in the first 30 days included in panel A. Survival curves after excluding patients with 90 -day mortality are shown in panel B. $p$ value from log-rank test is shown. The y-axis represents survival probability.

$81 \mathrm{~Gy}$, which is equivalent to 54 Gy in 30 fractions. However, that difference was not significant after correcting for multiple comparisons. A similar dosing was advocated by Dziuk et al., ${ }^{7}$ and was shown to improve progression-free survival. $^{8}$ In another report, a dose $>50$ Gy was associated with improved OS. ${ }^{11}$ A possible explanation of our finding is RT-related adverse events, which are more com- mon at higher doses-with a reported rate up to $16 \%$ in retrospective series. ${ }^{9}$ The prospective Radiation Therapy Oncology Group (RTOG) 0539 study uses similar dosing, and reported an adverse event rate of approximately $25 \%$ for intermediate-risk meningiomas-however, none of the adverse events were grade III. ${ }^{17}$ We only included photonbased RT modalities, which make it challenging to deliver 
higher doses safely. Several authors have advocated for proton-only or combined proton-photon RT for safe delivery of doses $>60 \mathrm{~Gy}$, which were shown to improve OS in AM. ${ }^{3,9}$ Chordoma is a similarly radioresistant and aggressive tumor, with current adoption of proton-based RT for adjuvant treatment showing improved efficacy at delivering the large RT doses required. ${ }^{21}$ We cannot exclude selection bias affecting this result, in which patients with more aggressive tumors or greater tumor burden were receiving higher doses of radiation.

Finally, we investigated factors associated with longterm survival. We found that age was associated with long-term survival at 10 years on univariate analysis. This confirms prior reports. ${ }^{11,16,18}$ Long-term survivors were 15 years younger on average, which may be a reflection of their medical status. However, the effect remained significant on multivariate analysis independent of comorbidity scores. Alternatively, the difference in survival might be due to different molecular genetic profiles in the two age groups similar to isocitrate dehydrogenase (IDH) mutation in glioblastoma. Recent work on genetic profiling of AM revealed two distinct subtypes with divergent clinical courses and with a slight age difference between the two groups. ${ }^{5}$ However, they did not include younger patients similar to our cohort.

\section{Limitations}

The NCDB reports initial courses of treatment and therefore lacks information regarding reoperation and salvage $\mathrm{RT}$, which can affect OS. Additionally, the NCDB reports all-cause mortality rather than cancer-specific deaths. However, no other database has similar long-term survival for as big a sample. Also, the NCDB does not include Simpson grading. However, no data have shown improved survival with Simpson grade 1. Finally, the NCDB is a retrospective database that also lacks radiological data and exact tumor locations. Ongoing prospective studies like the RTOG 0539 are needed to provide higher-level evidence.

\section{Conclusions}

Anaplastic meningiomas are aggressive tumors that carry a poor prognosis. Conventional adjuvant RT improves local control. However, adjuvant RT may not offer a long-term survival benefit. Photon-based RT doses higher than 54 Gy might be associated with decreased survival.

\section{References}

1. Aizer AA, Bi WL, Kandola MS, Lee EQ, Nayak L, Rinne ML, et al: Extent of resection and overall survival for patients with atypical and malignant meningioma. Cancer 121:43764381, 2015

2. Bilimoria KY, Stewart AK, Winchester DP, Ko CY: The National Cancer Data Base: a powerful initiative to improve cancer care in the United States. Ann Surg Oncol 15:683690, 2008

3. Boskos C, Feuvret L, Noel G, Habrand JL, Pommier P, Alapetite $\mathrm{C}$, et al: Combined proton and photon conformal radiotherapy for intracranial atypical and malignant meningioma. Int J Radiat Oncol Biol Phys 75:399-406, 2009
4. Charlson ME, Pompei P, Ales KL, MacKenzie CR: A new method of classifying prognostic comorbidity in longitudinal studies: development and validation. J Chronic Dis 40:373383, 1987

5. Collord G, Tarpey P, Kurbatova N, Martincorena I, Moran S, Castro M, et al: An integrated genomic analysis of anaplastic meningioma identifies prognostic molecular signatures. Sci Rep 8:13537, 2018

6. Durand A, Labrousse F, Jouvet A, Bauchet L, Kalamaridès $\mathrm{M}$, Menei P, et al: WHO grade II and III meningiomas: a study of prognostic factors. J Neurooncol 95:367-375, 2009

7. Dziuk TW, Woo S, Butler EB, Thornby J, Grossman R, Dennis WS, et al: Malignant meningioma: an indication for initial aggressive surgery and adjuvant radiotherapy. J Neurooncol 37:177-188, 1998

8. Goldsmith BJ, Wara WM, Wilson CB, Larson DA: Postoperative irradiation for subtotally resected meningiomas. A retrospective analysis of 140 patients treated from 1967 to 1990. J Neurosurg 80:195-201, 1994

9. Hug EB, Devries A, Thornton AF, Munzenride JE, Pardo FS, Hedley-Whyte ET, et al: Management of atypical and malignant meningiomas: role of high-dose, 3D-conformal radiation therapy. J Neurooncol 48:151-160, 2000

10. Kaur G, Sayegh ET, Larson A, Bloch O, Madden M, Sun MZ, et al: Adjuvant radiotherapy for atypical and malignant meningiomas: a systematic review. Neuro Oncol 16:628-636, 2014

11. Milosevic MF, Frost PJ, Laperriere NJ, Wong CS, Simpson WJ: Radiotherapy for atypical or malignant intracranial meningioma. Int J Radiat Oncol Biol Phys 34:817-822, 1996

12. Mohanty S, Bilimoria KY: Comparing national cancer registries: The National Cancer Data Base (NCDB) and the surveillance, epidemiology, and end results (SEER) program. J Surg Oncol 109:629-630, 2014

13. Orton A, Frandsen J, Jensen R, Shrieve DC, Suneja G: Anaplastic meningioma: an analysis of the National Cancer Database from 2004 to 2012. J Neurosurg 128:1684-1689, 2018

14. Ostrom QT, Gittleman H, Truitt G, Boscia A, Kruchko C, Barnholtz-Sloan JS: CBTRUS Statistical Report: Primary Brain and Other Central Nervous System Tumors Diagnosed in the United States in 2011-2015. Neuro Oncol 20 (Suppl 4):iv1-iv86, 2018

15. Park HS, Gross CP, Makarov DV, Yu JB: Immortal time bias: a frequently unrecognized threat to validity in the evaluation of postoperative radiotherapy. Int J Radiat Oncol Biol Phys 83:1365-1373, 2012

16. Pasquier D, Bijmolt S, Veninga T, Rezvoy N, Villa S, Krengli $\mathrm{M}$, et al: Atypical and malignant meningioma: outcome and prognostic factors in 119 irradiated patients. A multicenter, retrospective study of the Rare Cancer Network. Int J Radiat Oncol Biol Phys 71:1388-1393, 2008

17. Rogers L, Zhang P, Vogelbaum MA, Perry A, Ashby LS, Modi JM, et al: Intermediate-risk meningioma: initial outcomes from NRG Oncology RTOG 0539. J Neurosurg 129:35-47, 2018

18. Rosenberg LA, Prayson RA, Lee J, Reddy C, Chao ST, Barnett GH, et al: Long-term experience with World Health Organization grade III (malignant) meningiomas at a single institution. Int J Radiat Oncol Biol Phys 74:427-432, 2009

19. Stessin AM, Schwartz A, Judanin G, Pannullo SC, Boockvar JA, Schwartz TH, et al: Does adjuvant external-beam radiotherapy improve outcomes for nonbenign meningiomas? A Surveillance, Epidemiology, and End Results (SEER)-based analysis. J Neurosurg 117:669-675, 2012

20. Sughrue ME, Sanai N, Shangari G, Parsa AT, Berger MS, McDermott MW: Outcome and survival following primary and repeat surgery for World Health Organization Grade III meningiomas. J Neurosurg 113:202-209, 2010

21. Walcott BP, Nahed BV, Mohyeldin A, Coumans JVV, Kahle 
KT, Ferreira MJ: Chordoma: current concepts, management, and future directions. Lancet Oncol 13:e69-e76, 2012

22. Wang C, Kaprealian TB, Suh JH, Kubicky CD, Ciporen JN, Chen Y, et al: Overall survival benefit associated with adjuvant radiotherapy in WHO grade II meningioma. Neuro Oncol 19:1263-1270, 2017

23. Willis J, Smith C, Ironside JW, Erridge S, Whittle IR, Everington D: The accuracy of meningioma grading: a 10-year retrospective audit. Neuropathol Appl Neurobiol 31:141149,2005

24. Yang SY, Park CK, Park SH, Kim DG, Chung YS, Jung HW: Atypical and anaplastic meningiomas: prognostic implications of clinicopathological features. J Neurol Neurosurg Psychiatry 79:574-580, 2008

25. Zhao P, Hu M, Zhao M, Ren X, Jiang Z: Prognostic factors for patients with atypical or malignant meningiomas treated at a single center. Neurosurg Rev 38:101-107, 2015

\section{Disclosures}

Dr. Williams is a consultant for Monteris Medical.

\section{Author Contributions}

Conception and design: Williams, Alhourani, Aljuboori. Acquisition of data: Williams, Alhourani, Aljuboori. Analysis and interpretation of data: Williams, Alhourani. Drafting the article: Alhourani. Critically revising the article: Williams, Alhourani, Yusuf, Woo, Hattab, Andaluz. Reviewed submitted version of manuscript: all authors. Approved the final version of the manuscript on behalf of all authors: Williams. Statistical analysis: Alhourani. Study supervision: Woo.

\section{Correspondence}

Brian J. Williams: University of Louisville, School of Medicine, Louisville, KY. brian.williams@ulp.org. 